\title{
Ensemble Average of an Arbitrary Number of Pairs of Different Eigenvalues Using Grassmann Integration
}

\author{
Nazakat Ullah \\ Tata Institute of Fundamental Research, Homi Bhabha Road, Bombay 400005, India
}

\begin{abstract}
An identity satisfied by the eigenvalues of a real-symmetric matrix and an integral representation of a determinant using Grassmann variables are used to show that the ensemble average of $S$ different pairs of eigenvalues of a GOE is given by $(-1)^{S} 2^{-S} \pi^{-1 / 2} \Gamma\left(S+\frac{1}{2}\right)$.
\end{abstract}

\section{Introduction}

The idea of a matrix ensemble originally introduced by Wigner [1] has been successfully used to study the average properties of the compound nucleus levels [2]. The basic assumption is that each element of a real-symmetric $N \times N$ Hamiltonian matrix has an independent Gaussian distribution. This leads to an eigenvalue distribution known as the Wishart distribution. Because of the appearance of a factor with absolute sign in this distribution, further integrations [3] over the eigenvalues cannot be carried out in a simple way. The purpose of the present work is to show that a simple identity between the eigenvalues and the matrix elements of the Hamiltonian together with a representation of a determinant in terms of Grassmann variables can be used to find ensemble averages of the products of eigenvalues. We shall describe this formulation in Sect. 2. The concluding remarks will be presented in Sect. 3 .

\section{Formulation}

Let us consider a real-symmetric $N \times N$ Hamiltonian matrix, then the joint distribution of its $N$ diagonal and $\frac{1}{2} N(N-1)$ off-diagonal matrix elements can be written as

$$
P\left(\left\{H_{i j}\right\}\right)=2^{\frac{1}{4} N(N-1)} \pi^{\frac{1}{4} N(N+1)} \exp \left(-\operatorname{Tr} H^{2}\right) .
$$

The eigenvalues $E_{i}$ of the Hamiltonian matrix $H$ satisfy the following identity:

where $\lambda$ is a parameter.

$$
\prod_{i=1}^{N}\left(1-\lambda E_{i}\right)=\operatorname{det}(1-\lambda H)
$$


Taking the ensemble average of (2) we can write

$$
\left\langle\prod_{i}\left(1-\lambda E_{i}\right)\right\rangle=\langle\operatorname{det}(1-\lambda H)\rangle \text {. }
$$

We now use the Grassmann representation of a determinant given by [4],

$$
\operatorname{det}(1-\lambda H)=\int \exp -\sum a_{m}^{*}\left(\delta_{m n}-\lambda H_{m n}\right) a_{n} \Pi d a_{m}^{*} d a_{m},
$$

and carry out the integration over the variables $H_{i j}$ using the distribution given by expression (1). This gives us

$$
\left\langle\prod_{i}\left(1-\lambda E_{i}\right)\right\rangle=\int \exp \left[-\sum a_{\mu}^{*} a_{\mu}-\frac{\lambda^{2}}{4} \sum_{\mu<v}\left(a_{\mu}^{*} a_{\mu} a_{v}^{*} a_{v}\right)\right] \Pi d a_{\mu}^{*} d a_{\mu} .
$$

Writing

$$
\sum_{\mu<v} a_{\mu}^{*} a_{\mu} a_{\nu}^{*} a_{v}=\frac{1}{2}\left(\sum_{\mu} a_{\mu}^{*} a_{\mu}\right)^{2}
$$

and using a transformation which converts a Gaussian into an exponential, we can rewrite expression (5) as

$$
\left.\left\langle\prod_{i}\left(1-\lambda E_{i}\right)\right\rangle=\pi^{-\frac{1}{2}} \int_{-\infty}^{\infty} d t \exp \left(-t^{2}\right) \int \exp -\left(1-i \frac{\sqrt{2}}{2} \lambda t\right) \sum a_{\mu}^{*} a_{\mu}\right) \Pi d a_{\mu}^{*} d a_{\mu} .
$$

Carrying out the integrations over Grassmann variables and the integration over $t$ we get

$$
\left\langle\prod_{i}\left(1-\lambda E_{i}\right)\right\rangle=\sum_{S=0} \frac{N !}{(N-2 S) ! S !} \frac{(-1)^{S}}{2^{3 S}} \lambda^{2 S},
$$

where the summation over $S$ goes from 0 to the nearest integer less than or equal to $\frac{N}{2}$.

Equating powers of $\lambda$ on both sides in (8), we finally arrive at the desired result:

$$
\left\langle E_{1} E_{2} \ldots E_{2 S}\right\rangle=(-1)^{S} 2^{-S} \pi^{-1 / 2} \Gamma\left(S+\frac{1}{2}\right) .
$$

\section{Conclusions}

We have shown how one can obtain ensemble averages of the products of eigenvalues using Grassmann integration. It is obvious from expression (8) or the Wishart distribution itself that the ensemble average of an odd number of eigenvalues is zero. Recursive relations for the ensemble averages of products of eigenvalues have been given in [5] by writing the product on the left-hand side of identity (2) in two different ways. Lastly we remark that the ensemble average given by expression (9) was conjectured [6] earlier using the results for one and two pairs of different eigenvalues. 


\section{References}

1. Wigner, E.P.: Statistical properties of real symmetric matrices with many dimensions. Can. Math. Cong. Proc. Toronto, Canada: University of Toronto Press 1957

2. Mahaux, C., Weidenmüller, H.A.: Shell model approach to nuclear reactions. Amsterdam: North-Holland 1969

3. Mehta, M.L.: Random matrices. New York: Academic Press 1967

4. Balian, R., Zinn-Justin, J. (eds.): Methods in field theory. Les Houches Ecole d'Etude Physique Théorique, Session XXVIII. Amsterdam: North-Holland 1976

5. Verbaarschot, J.J.M., Weidenmüller, H.A., Zirnbauer, M.R.: Evaluation of ensemble averages for simple Hamiltonians perturbed by a GOE interaction. Ann. Phys. 153, 367-388 (1984)

6. Ullah, N.: Invariance hypothesis and higher correlations of Hamiltonian matrix elements. Nucl. Phys. 58, 65-71 (1964)

Communicated by H. Araki

Received November 2, 1985 
